\title{
Combination therapy with gemcitabine and nab-paclitaxel for locally advanced unresectable pancreatic cancer
}

\author{
TAKESHI SAITO, KEINOSUKE ISHIDO, DAISUKE KUDO, NORIHISA KIMURA, \\ TAIICHI WAKIYA, YOSHIHITO NAKAYAMA and KENICHI HAKAMADA \\ Department of Gastroenterological Surgery, Hirosaki University Graduate \\ School of Medicine, Hirosaki, Aomori 036-8562, Japan
}

Received January 26, 2017; Accepted March 24, 2017

DOI: $10.3892 / \mathrm{mco} .2017 .1251$

\begin{abstract}
The aim of the present study was to investigate the early treatment outcomes of combined gemcitabine and nab-paclitaxel treatment for locally advanced unresectable pancreatic cancer (LURPC). The subjects comprised 7 patients with LURPC receiving the abovementioned combination therapy at the Hirosaki University Hospital (Hirosaki, Japan) between January and September, 2015. The clinicopathological factors, adverse events and response to treatment were investigated. To determine whether the cases were unresectable, the National Comprehensive Cancer Network guidelines, version 2. 201,) were applied. The patients underwent a median of 4 (range, 2-7) courses of treatment. The response to treatment was evaluated using the Response Evaluation Criteria In Solid Tumors. The subjects included 1 male and 6 female LURPC patients, with a median age of 71 years (range, 59-78 years). The tumor was located in the head and body of the pancreas in 6 and 1 patients, respectively. No patients achieved a complete response, 5 achieved a partial response, 2 had stable disease, and none exhibited progressive disease. The response rate was $71 \%$. The mean tumor diameter decreased significantly from $35 \mathrm{~mm}$ (range, 24-60 mm) prior to treatment to $22 \mathrm{~mm}$ (range, 20-35 mm) following treatment. Two patients were downstaged. The mean carbohydrate antigen (CA) 19-9 values decreased significantly from $767 \mathrm{U} / \mathrm{ml}$ (range, 14-1,977 U/ml) prior to treatment to $35 \mathrm{U} / \mathrm{ml}$ (range, 14-123 U/ml) following treatment. Adverse events classified as grade $\geq 3$ occurred in 4 patients (57\%): 3 patients (43\%) suffered from neutropenia and 1 patient (14\%) developed bilateral cellulitis of the lower extremities. No patients experienced an increase in disease severity, and all were able to continue treatment following temporary withdrawal or dosage reduction. Therefore, combined treatment with
\end{abstract}

Correspondence to: $\mathrm{Dr}$ Takeshi Saito, Department of Gastroenterological Surgery, Hirosaki University Graduate School of Medicine, 5 Zaifutyo, Hirosaki, Aomori 036-8562, Japan E-mail: tt83fe@bma.biglobe.ne.jp

Key words: combination therapy, gemcitabine, nab-paclitaxel, pancreatic cancer, unresectable gemcitabine and nab-paclitaxel had favorable tumor-reducing effects and was not associated with severe adverse events, suggesting that this is a useful therapeutic strategy for patients with LURPC.

\section{Introduction}

Pancreatic cancer (PC) has an extremely high degree of malignancy, and the number of affected patients is increasing annually (1). Currently, PC is the fourth highest cause of cancer-related mortality among US adults and the fifth and fourth leading cause of death among Japanese men and women, respectively $(1,2)$. Resection is the only form of treatment associated with a complete cure, but only $10-20 \%$ of cases are resectable and the majority of the cases involve metastatic or locally advanced unresectable PC (LURPC) (3). LURPC accounts for 30-35\% of all PC cases (4), and its treatment options include chemotherapy or chemoradiotherapy (CRT). However, its prognosis remains poor, and there is an urgent need for novel treatment methods (5).

The recently conducted MPACT trial verified that a combination therapy comprising gemcitabine (GEM) and nab-paclitaxel (nab-PTX) significantly prolonged the survival of patients with metastatic PC compared with a therapy comprising GEM alone (6). The results of that study indicated that this regimen may become a new therapeutic option. However, the responses to this form of treatment for LURPC have not yet been fully elucidated. The aim of the present study was to investigate the safety and efficacy of the combination of GEM and nab-PTX for the treatment of patients with LURPC.

\section{Patients and methods}

Patients. A total of 7 patients with LURPC were treated with a combination regimen comprising GEM and nab-PTX at the Department of Gastroenterological Surgery of the Hirosaki University Hospital (Hirosaki, Japan) between January and September, 2015. The chemotherapy regimen included the administration of GEM $\left(1,000 \mathrm{mg} / \mathrm{m}^{2}\right.$ on days 1,8 and 15 , every 4 weeks) and nab-PTX ( $125 \mathrm{mg} / \mathrm{m}^{2}$ on days 1,8 and 15 , every 4 weeks). Resectability was determined according to the National Comprehensive Cancer Network (NCCN) guidelines, version 2. 2015 (7). The number of chemotherapy courses, 
rate of change in tumor diameter, rate of change of serum carbohydrate CA19-9 values, incidence of grade $\geq 3$ adverse events, therapeutic effects and survival time were investigated in all the cases. In an effort to determine specific response to treatment, the Response Evaluation Criteria In Solid Tumors guidelines, version 1.1 (8) were used for analyzing patient computed tomography images. Survival time was defined as the period from the date of treatment initiation to the date on which the outcome was achieved. Overall patient survival was analyzed using the Kaplan-Meier method. All analyses were performed using the IBM SPSS ${ }^{\circledR}$ Statistics version 24.0 for Windows (IBM, Armonk, NY, USA). This study was approved by the Human Research Ethics Committee of Hirosaki University (no. 2016-1038) and informed consent was provided by all the participants.

\section{Results}

Patient characteristics. The patient characteristics are summarized in Table I. The median patient age was 71 years (range, 59-78 years), and the subjects comprised 1 male and 6 female LURPC patients. The tumor was located at the body of the pancreas in 1 and in the head of the pancreas in 6 subjects. The criteria for diagnosing a case as unresectable were as follows: Solid tumor in contact with the celiac axis $>180^{\circ}$ in 3 subjects, solid tumor in contact with the common hepatic artery with extension to the hepatic artery bifurcation in 2 subjects, an unreconstructible portal vein due to tumor involvement in 1 subject, and an unreconstructible portal vein/superior mesenteric vein involvement in 1 subject. The patients underwent a median of 4 (range, 2-7) courses of chemotherapy. No patients achieved a complete response, 5 achieved a partial response, 2 had stable disease, and none had progressive disease. The response rate was $71 \%$ (Fig. 1).

Therapeutic effects. The therapeutic effects of the combination treatment are summarized in Table II. The median tumor diameter significantly decreased from $35 \mathrm{~mm}$ (range, 24-60 mm) prior to treatment to $22 \mathrm{~mm}$ (range, $20-35 \mathrm{~mm}$ ) following treatment $(\mathrm{P}=0.008)$; the median rate of tumor shrinkage was $37 \%$ (range, 0-57\%). The mean serum CA19-9 values significantly decreased from $767 \mathrm{U} / \mathrm{ml}(14-1977 \mathrm{U} / \mathrm{ml})$ prior to treatment to $35 \mathrm{U} / \mathrm{ml}$ (range, 14-123 U/ml) following treatment $(\mathrm{P}=0.038)$; the median rate of reduction was $92 \%$ (range, $47-98 \%$ ). When the 5 subjects who achieved a partial response were examined, 2 were diagnosed with resectable cancer and both underwent radical $\mathrm{R} 0$ resection. Peritoneal dissemination was observed in 1 patient 2 months after surgery; despite initiating treatment, the patient succumbed to the disease 1 month later. Another patient was found to be recurrence-free during the 3-month follow-up, and S-1 was administered as ongoing postoperative adjuvant chemotherapy. The mean survival time for the 7 patients was 13.3 months [95\% confidence interval (CI): 11.3-15.3; Fig. 2].

Adverse events classified as grade $\geq 3$ occurred in 4 patients (57\%): 3 patients (43\%) suffered from neutropenia and $1(14 \%)$ developed bilateral cellulitis of the lower extremities. None of these patients experienced an increase in disease severity, and all were able to continue treatment following temporary withdrawal or dosage reduction.

\section{Discussion}

The nab-PTX formulation comprises human albumin-bound PTX nanoparticles and has been reported to be useful for treating breast, gastric and non-small-cell lung cancer (9-11). When combined with GEM for the treatment of PC, nab-PTX acts by decreasing the interstitial components of $\mathrm{PC}$ and increases the microvasculature within the tumor. By doing so, the intratumoral concentration of GEM is increased by $\sim 3$-fold (12). In addition, nab-PTX decreases the plasma concentration of cytidine deaminase, one of the enzymes that metabolizes GEM, and has also been reported to stabilize the active form of an intratumoral GEM metabolite, gemcitabine triphosphate (13). Therefore, the direct anticancer and synergistic effects of nab-PTX used in combination with GEM enable powerful tumor-reducing effects. During the MPACT trial, the combination therapy comprising GEM and nab-PTX was more efficient in prolonging patient survival compared with GEM alone for the treatment of metastatic PC (8.5 months; hazard ratio=0.72) (6) and has also been shown to be useful in phase II/III clinical trials in Japan [median progression-free survival: 6.5 months (95\% CI: 5.1-8.3); median overall survival: 13.5 months (95\% CI: 10.6-not reached)] (14). However, these reports investigated patients with metastatic $\mathrm{PC}$, and this regimen remains to be investigated using patients with LURPC as subjects. Accordingly, in the present study, the safety and efficacy of a combination therapy comprising GEM and nab-PTX for the treatment of patients with LURPC were investigated. The median tumor regression rate was $37 \%$ (range, $0-57 \%$ ), and powerful tumor-reducing effects were achieved. Furthermore, the median survival time was 13.3 months, which is considered to be a favorable outcome.

The NCCN guidelines recommend chemotherapy or CRT for the treatment of LURPC when the performance status of the patients is favorable. To date, chemotherapy using GEM, 5-fluorouracil (5-FU)/leucovorin (LV) or capecitabine, or combined treatment comprising radiation in the form of CRT, are recommended. In recent years, 5-FU/LV plus oxaliplatin and irinotecan (FOLFIRINOX regimen) have been reported to be useful and recommended as a new treatment regimen. Recent reports regarding the treatment of LURPC are presented in Table III. Habermehl et al investigated CRT using GEM for the treatment of LURPC and reported a response rate (RR) of $9 \%$, with a median survival time of 12.3 months (15). In addition, Faris et al evaluated the FOLFIRINOX regimen for the treatment of LURPC and found an RR of $27 \%$, as well as favorable treatment outcomes (median disease-free survival: 11.3 months); however, the median survival time was not reported (3). Moreover, Nanda and Blazer et al also reported favorable outcomes when they compared FOLFIRINOX with CRT for the treatment of LURPC, with median survival times of 18.6 and 12.2 months, respectively $(16,17)$. During the present study, the RR was $71 \%$, and although the median survival time was not calculated, the mean survival time was 13.3 months. These findings suggest that the combination therapy comprising GEM and nab-PTX is effective for treating patients with LURPC.

There are reports of severe adverse events during chemotherapy and CRT treatment for LURPC. Chen et al performed a meta-analysis comparing CRT, chemotherapy alone and 
Table I. Characteristics of patients with locally advanced unresectable pancreatic cancer.

\begin{tabular}{|c|c|c|c|c|c|c|}
\hline No. & Age, years & Gender & Location & $\begin{array}{l}\text { Factor determining } \\
\text { unresectability }\end{array}$ & $\begin{array}{c}\text { Number of } \\
\text { chemotherapy cycles }\end{array}$ & RECIST \\
\hline 1 & 74 & $\mathrm{~F}$ & Head & Solid tumor contact with $\mathrm{CA}>180^{\circ}$ & 7 & PR \\
\hline 2 & 71 & $\mathrm{~F}$ & Head & Unreconstructible PV due to tumor involvement & 5 & $\mathrm{SD}$ \\
\hline 3 & 66 & M & Head & $\begin{array}{l}\text { Solid tumor contact with CHA with extension } \\
\text { to hepatic artery bifurcation }\end{array}$ & 2 & PR \\
\hline 4 & 59 & $\mathrm{~F}$ & Head & Solid tumor contact with $\mathrm{CA}>180^{\circ}$ & 4 & PR \\
\hline 5 & 65 & $\mathrm{~F}$ & Body & Solid tumor contact with $\mathrm{CA}>180^{\circ}$ & 2 & $\mathrm{SD}$ \\
\hline 6 & 78 & $\mathrm{~F}$ & Head & $\begin{array}{l}\text { Unreconstructible PV/SMV due to } \\
\text { tumor involvement }\end{array}$ & 4 & PR \\
\hline 7 & 77 & $\mathrm{~F}$ & Head & $\begin{array}{l}\text { Solid tumor contact with CHA with } \\
\text { extension to the hepatic artery bifurcation }\end{array}$ & 2 & PR \\
\hline
\end{tabular}

F, female; M, male; CS; conversion surgery; CA, celiac axis; CHA, common hepatic artery; PV, portal vein; SMV, superior mesenteric vein; PR, partial response; SD, stable disease; RECIST, Response Evaluation Criteria In Solid Tumors.

Table II. Evaluation of the chemotherapy for locally advanced unresectable pancreatic cancer.

\begin{tabular}{|c|c|}
\hline Variables & Values \\
\hline \multicolumn{2}{|c|}{ Tumor diameter, mm [median (range)] } \\
\hline Pre CTx & $35(24-60)$ \\
\hline Post CTx & $20(15-35)$ \\
\hline Reduction rate $(\%)$ & $37(0-57)$ \\
\hline \multicolumn{2}{|l|}{ CA19-9, U/ml } \\
\hline Pre CTx & $247(14-1,977)$ \\
\hline Post CTx & $35(4-123)$ \\
\hline Reduction rate $(\%)$ & $92(47-98)$ \\
\hline $\mathrm{RR}, \%$ & $\begin{array}{c}71(\mathrm{CR}, 0 ; \mathrm{PR}, 5 \\
\quad \mathrm{SD}, 2 ; \mathrm{PD}, 0)\end{array}$ \\
\hline \multicolumn{2}{|c|}{ Grade $>3$ adverse events, n (\%) } \\
\hline Neutropenia & $3(43)$ \\
\hline Cellulitis & $1(14)$ \\
\hline CS & $2(29)$ \\
\hline $\mathrm{R} 0$ resection, $\mathrm{n} /$ total $(\%)$ & $2 / 2(100)$ \\
\hline
\end{tabular}

RR, response rate; CS, conversion surgery; CTx, chemotherapy; CR, complete response; $\mathrm{PR}$, partial response; $\mathrm{SD}$, stable disease; $\mathrm{PD}$, progressive disease.

radiotherapy alone for LURPC, and reported that grade $\geq 3$ adverse events were common (18). In addition, a phase III trial (FFCD-SFRO trial) compared CRT using 5-FU and cisplatin to chemotherapy using GEM alone. They found that, compared with the chemotherapy group, the CRT group was associated with a significantly higher incidence of hematotoxic and non-hematotoxic adverse events, such as infection, vomiting and diarrhea (66 vs. $40 \%$, respectively; $\mathrm{P}=0.008$ ) (19). The same trial also observed a significant prolongation of median survival time in the chemotherapy group (13.0 vs. 8.6 months; $\mathrm{P}=0.03)$, suggesting that chemotherapy is useful for treating LURPC (19).

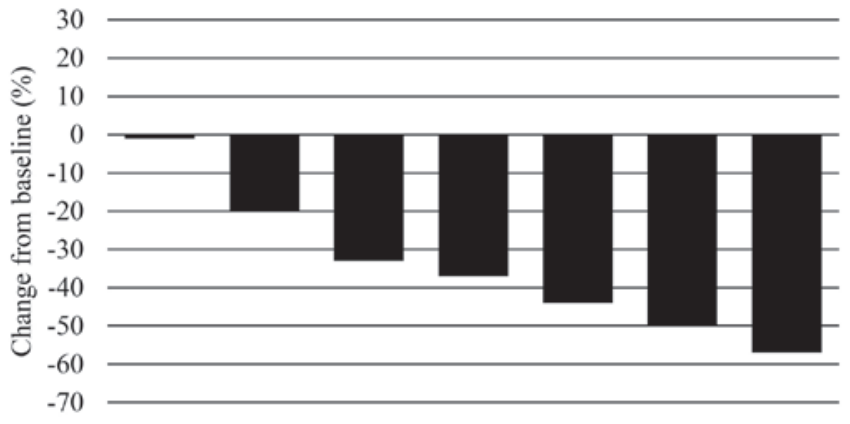

Figure 1. Waterfall plot of maximum percentage changes from baseline in the size of target lesions according to the Response Evaluation Criteria In Solid Tumors $(\mathrm{n}=7)$. A total of 5 patients exhibited partial response $(71 \%)$ and 2 patients had stable disease $(29 \%)$.

Moreover, another phase III trial (ECOG-4201 trial) compared CRT using GEM alone to chemotherapy using GEM alone. There were no significant differences in the incidence of grade $\geq 3$ adverse events ( 79 vs. $77 \%$, respectively; $\mathrm{P}=0.1$ ) and the median survival time was reported to be significantly longer in the CRT group (11.1 vs. 9.2 months, respectively; $\mathrm{P}=0.017$ ) (20). There continues to be a multitude of such contradictory reports and discussion regarding the usefulness of CRT for the treatment of LURPC (21). In terms of using FOLFIRINOX to treat LURPC and metastatic PC, although some reports have described a high response to treatment, with RRs 9-27\%, other studies have reported the presence of grade $\geq 3$ adverse events, including neutropenia (46\%), loss of appetite (23.6\%), vomiting $(14.5 \%)$, diarrhea (13\%) and peripheral neuropathy (9\%) (22-24). Furthermore, reports on metastatic PC have investigated the toxicity of the combination therapy comprising GEM and nab-PTX and found grade $\geq 3$ adverse events such as neutropenia (38\%), loss of appetite (17\%), peripheral neuropathy (17\%) and diarrhea (6\%) (6). Although the frequency of peripheral neuropathy is marginally higher with the combination therapy, the overall incidence of adverse events is lower compared with that of FOLFIRINOX. In addition, $8 \%$ of the subjects were unable to continue the study due to peripheral neuropathy, and 
Table III. Previous studies on chemotherapy for locally advanced unresectable pancreatic cancer.

\begin{tabular}{llccccccc}
\hline No. & First author & Year & Patient no. & Regimen & RR $(\%)$ & CS $(\%) /$ R0 resection (\%) & MST (months) & (Refs.) \\
\hline 1 & Habermehl & 2012 & 198 & GEM/radiation & 9 & $26 / 39$ & 12.3 & $(15)$ \\
2 & Faris & 2013 & 22 & FOLFIRINOX & 27.3 & $22 / 60$ & NA & $(3)$ \\
3 & Nanda & 2015 & 15 & FOLFIRINOX & NA & $13 /$ NA & 18.6 & $(16)$ \\
4 & Blazer & 2015 & 25 & FOLFIRINOX & 9 & $44 / 91$ & 21.2 & $(17)$ \\
5 & Present study & 2015 & 7 & GEM/nab-PTX & 71 & $29 / 100$ & (mean) 13.3 & \\
\hline
\end{tabular}

GEM, gemcitabine; FOLFIRINOX, 5- fluorouracil/leucovorin plus oxaliplatin and irinotecan; nab-PTX, nab-paclitaxel; RR, response rate; CS, conversion surgery; MST, median survival time; NA, not applicable.

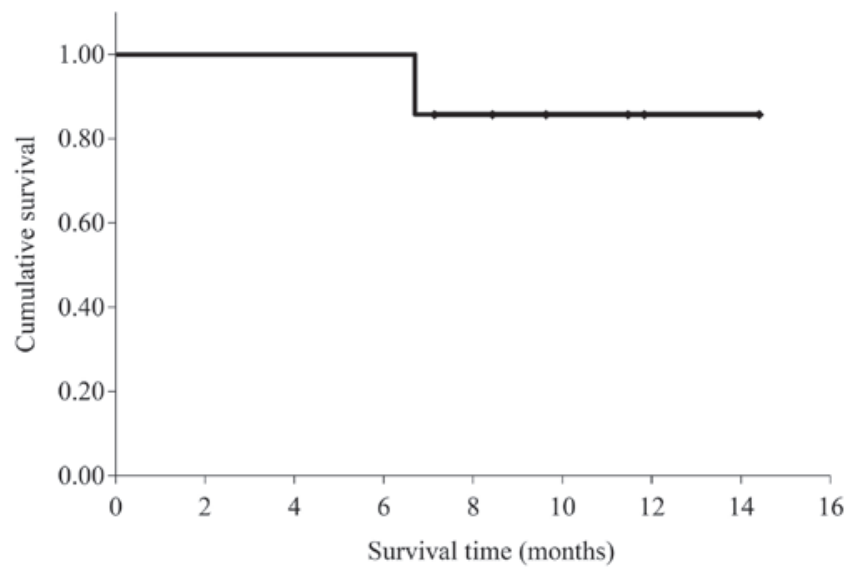

Figure 2. Kaplan-Meier survival curve for overall survival. The mean overall survival was 13.3 months (95\% confidence interval: $11.3-15.3)$.

approximately half of them improved to grade $\leq 1$ following dosage reduction (6). During the present study, grade 3 adverse events were observed in 4 of the 7 subjects (neutropenia in 3 and cellulitis in 1 subjects); however, these events were mild and did not increase in severity during the clinical course in any of the 4 subjects. All the patients were able to continue the study on an outpatient basis following temporary treatment withdrawal or dosage reduction. Combination therapy comprising GEM and nab-PTX is associated with a lower incidence of adverse events compared with CRT and FOLFIRINOX. Furthermore, as a high quality of life was maintained during our treatment, we consider this treatment to be a highly favorable option.

If tumors become resectable following a successful primary course of treatment, conversion surgery (CS) may prolong survival. A study by Satoi et al compared a group that underwent CS following primary treatment for LURPC to a group that did not, and found that the median survival was significantly prolonged in the CS group (CS vs. non-CS: 39.7 vs. 20.8 months, respectively; $\mathrm{P}<0.001)(25)$. Similarly, Habermehl et al reported that median survival was significantly prolonged in the CS group (CS vs. non-CS: 11.9 vs. 14.4 months, respectively; $\mathrm{P}=0.004)(15)$; they also reported an even longer patient survival if the surgical margins were found to be clear (R0 vs. R1 vs. R2: 22.1 vs. 15.6 vs. 10.3 months, respectively) (15). Accordingly, it is extremely important to develop treatment methods that incorporate CS, which is a highly successful treatment strategy for LURPC. As shown in Table III, when Habermehl et al investigated CRT as a treatment for LURPC, CS was performed in $26 \%$ of the cases and the rate of clear surgical margins was $39 \%$ (15). When Faris et al studied the FOLFIRINOX regimen for treating LURPC, they reported that CS was performed in $22 \%$ of the cases and the rate of clear surgical margins was $60 \%$, indicating more favorable treatment outcomes compared with those observed using CRT (3). In the present study, CS was performed in $29 \%$ of the cases and the rate of clear surgical margins was $100 \%$. Thus, combination therapy comprising GEM and nab-PTX is a potential treatment option associated with a higher CS rate, a higher rate of clear surgical margins, and a longer survival time compared with those observed with CRT or FOLFIRINOX.

Based on the abovementioned findings, this combination therapy is associated with a low incidence of adverse events, maintains the quality of life and markedly prolongs survival for patients with LURPC; thus, we consider it to be a useful form of treatment for such cases. CS is common during LURPC and should be considered as an important and novel treatment strategy. However, the present study was associated with the following limitations: i) The patient sample was very small, ii) the study design was retrospective and iii) the period of observation was brief. Thus, the usefulness of the combination therapy comprising GEM and nab-PTX for patients with LURPC requires verification by future prospective studies.

\section{Acknowledgements}

The authors would like to thank Crimson Interactive Pvt. Ltd. (Ulatus)-www.ulatus.jp for their assistance with the manuscript translation and editing.

\section{References}

1. Statistics and Information Department: Vital statistics 2014. Tokyo: Ministry of Health, Labor and Welfare, 2014. http://www. mhlw.go.jp/toukei/s aikin/hw/jinkou/kakutei14/index.html. Accessed December 20, 2016.

2. Siegel R, Ma J, Zou Z and Jemal A: Cancer statistics, 2014. CA Cancer J Clin 64: 9-29, 2014.

3. Faris JE, Blaszkowsky LS, McDermott S, Guimaraes AR, Szymonifka J, Huynh MA, Ferrone CR, Wargo JA, Allen JN, Dias LE, et al: FOLFIRINOX in locally advanced pancreatic cancer: The massachusetts general hospital cancer center experience. Oncologist 18: 543-548, 2013.

4. Philip PA: Locally advanced pancreatic cancer: Where should we go from here? J Clin Oncol 29: 4066-4068, 2011. 
5. Vincent A, Herman J, Schulick R, Hruban RH and Goggins M: Pancreatic cancer. Lancet 378: 607-620, 2011.

6. Von Hoff DD, Ervin T, Arena FP, Chiorean EG, Infante J, Moore M, Seay T, Tjulandin SA, Ma WW, Saleh MN, et al Increased survival in pancreatic cancer with nab-paclitaxel plus gemcitabine. N Engl J Med 369: 1691-1703, 2013.

7. National Comprehensive Cancer Network. NCCN clinical practic guideline in oncology: Pancreatic adenocarcinoma. Fort Washington, PA: National Comprehensive Cancer Network, Version 2: 22, 2015.

8. Eisenhauer EA, Therasse P, Bogaerts J, Schwartz LH, Sargent D, Ford R, Dancey J, Arbuck S, Gwyther S, Mooney M, et al: New response evaluation criteria in solid tumours: Revised RECIST guideline (version 1.1). Eur J Cancer 45: 228-247, 2009.

9. Nakayama N, Ishido K, Chin K, Nishimura K, Azuma M, Matsusaka S, Inokuchi Y, Tanabe S, Kumekawa Y and Koizumi W: A phase I study of S-1 in combination with nab-paclitaxel in patients with unresectable or recurrent gastric cancer. Gastric Cancer 20: 350-357, 2017

10. Gradishar WJ, Tjulandin S, Davidson N, Shaw H, Desai N, Bhar P, Hawkins M and O'Shaughnessy J: Phase III trial of nanoparticle albumin-bound paclitaxel compared with polyethylated castor oil-based paclitaxel in women with breast cancer. J Clin Oncol 23: 7794-7803, 2005.

11. Rizvi NA, Riely GJ, Azzoli CG, Miller VA, Ng KK, Fiore J, Chia G, Brower M, Heelan R, Hawkins MJ and Kris MG: Phase I/II trial of weekly intravenous 130-nm albumin-bound paclitaxel as initial chemotherapy in patients with stage IV non-small-cell lung cancer. J Clin Oncol 26: 639-643, 2008.

12. Alvarez R, Musteanu M, Garcia-Garcia E, Lopez-Casas PP, Megias D, Guerra C, Muñoz M, Quijano Y, Cubillo A, Rodriguez-Pascual J, et al: Stromal disrupting effects of nab-paclitaxel in pancreatic cancer. Br J Cancer 109: 926-933, 2013.

13. Frese KK, Neesse A, Cook N, Bapiro TE, Lolkema MP, Jodrell DI and Tuveson DA: nab-Paclitaxel potentiates gemcitabine activity by reducing cytidine deaminase levels in a mouse model of pancreatic cancer. Cancer Discov 2: 260-269, 2012.

14. Ueno H, Ikeda M, Ueno M, Mizuno N, Ioka T, Omuro Y Nakajima TE and Furuse J: Phase I/II study of nab-paclitaxel plus gemcitabine for chemotherapy-naive Japanese patients with metastatic pancreatic cancer. Cancer Chemother Pharmacol 77: 595-603, 2016

15. Habermehl D, Kessel K, Welzel T, Hof H, Abdollahi A, Bergmann F, Rieken S, Weitz J, Werner J, Schirmacher P, et al: Neoadjuvant chemoradiation with Gemcitabine for locally advanced pancreatic cancer. Radiat Oncol 7: 28, 2012.

16. Nanda RH, El-Rayes B, Maithel SK and Landry J: Neoadjuvant modified FOLFIRINOX and chemoradiation therapy for locally advanced pancreatic cancer improves resectability. J Surg Oncol 111: 1028-1034, 2015.

17. Blazer M, Wu C, Goldberg RM, Phillips G, Schmidt C, Muscarella P, Wuthrick E, Williams TM, Reardon J, Ellison EC, et al: Neoadjuvant modified (m) FOLFIRINOX for locally advanced unresectable (LAPC) and borderline resectable (BRPC) adenocarcinoma of the pancreas. Ann Surg Oncol 22: $1153-1159,2015$
18. Chen Y, Sun XJ, Jiang TH and Mao AW: Combined radiochemotherapy in patients with locally advanced pancreatic cancer: A meta-analysis. World J Gastroenterol 19: 7461-7471, 2013.

19. Chauffert B, Mornex F, Bonnetain F, Rougier P, Mariette C, Bouché O, Bosset JF, Aparicio T, Mineur L, Azzedine A, et al: Phase III trial comparing intensive induction chemoradiotherapy (60 Gy, infusional 5-FU and intermittent cisplatin) followed by maintenance gemcitabine with gemcitabine alone for locally advanced unresectable pancreatic cancer. Definitive results of the 2000-01 FFCD/SFRO study. Ann Oncol 19: 1592-1599, 2008.

20. Loehrer PJ Sr, Feng Y, Cardenes H, Wagner L, Brell JM, Cella D, Flynn P, Ramanathan RK, Crane CH, Alberts SR and Benson AB III: Gemcitabine alone versus gemcitabine plus radiotherapy in patients with locally advanced pancreatic cancer: An Eastern Cooperative Oncology Group trial. J Clin Oncol 29: 4105-4112, 2011.

21. Kim R and Salf MW: Is there an optimal neoadjuvant therapy for locally advanced pancreatic cancer? JOP 8: 279-288, 2007.

22. Conroy T, Paillot B, François E, Bugat R, Jacob JH, Stein U, Nasca S, Metges JP, Rixe O, Michel P, et al: Irinotecan plus oxaliplatin and leucovorin-modulated fluorouracil in advanced pancreatic cancer-a Groupe Tumeurs Digestives of the Federation Nationale des Centres de Lutte Contre le Cancer study. J Clin Oncol 23: 1228-1236, 2005.

23. Gourgou-Bourgade S, Bascoul-Mollevi C, Desseigne F, Ychou M, Bouché O, Guimbaud R, Bécouarn Y, Adenis A, Raoul JL, Boige V, et al: Impact of FOLFIRINOX compared with gemcitabine on quality of life in patients with metastatic pancreatic cancer: Results from the PRODIGE 4/ACCORD 11 randomized trial. J Clin Oncol 31: 23-29, 2013.

24. Conroy T, Desseigne F, Ychou M, Bouché O, Guimbaud R, Bécouarn Y, Adenis A, Raoul JL, Gourgou-Bourgade S, de la Fouchardière $\mathrm{C}$, et al: FOLFIRINOX versus gemcitabine for metastatic pancreatic cancer. N Engl J Med 364: 1817-1825, 2011

25. Satoi S, Yamaue H, Kato K, Takahashi S, Hirono S, Takeda S, Eguchi H, Sho M, Wada K, Shinchi H, et al: Role of adjuvant surgery for patients with initially unresectable pancreatic cancer with a long-term favorable response to non-surgical anti-cancer treatments: Results of a project study for pancreatic surgery by the Japanese Society of Hepato-Biliary-Pancreatic Surgery. J Hepatobiliary Pancreat Sci 20: 590-600, 2013. 